\title{
Use of a healing abutment-type scan body and closed-mouth impression technique for mandibular implant-supported fixed complete denture: a pilot case report
}

\author{
Ju-Mi Park', Yeon-Hee Park², and Jung-Jin Lee ${ }^{3 \star}$
}

${ }^{1}$ Professor, Department of Prosthodontics, Institute of Oral Bio-Science, School of Dentistry, Jeonbuk National University and Research Institute of Clinical Medicine of Jeonbuk National University-Biomedical Research Institute of Jeonbuk National University Hospital, Jeonju, Republic of Korea

${ }^{2}$ Fellow, Department of Prosthodontics, Institute of Oral Bio-Science, School of Dentistry, Jeonbuk National University and Research Institute of Clinical Medicine of Jeonbuk National University-Biomedical Research Institute of Jeonbuk National University Hospital, Jeonju, Republic of Korea

${ }^{3}$ Associated Professor, Department of Prosthodontics, Institute of Oral Bio-Science, School of Dentistry, Jeonbuk National University and Research Institute of Clinical Medicine of Jeonbuk National University-Biomedical Research Institute of Jeonbuk National University Hospital, Jeonju, Republic of Korea

\begin{abstract}
Numerous clinical and laboratory procedures are required for fabricating an implant-supported fixed complete denture. Such processes can be inconvenient for both dentists and patients, and errors can occur yielding adverse effects on the accuracy of the prosthesis. The introduction of digital technology as well as computer-aided design and manufacturing has allowed for the efficient fabrication of more accurate prostheses. In the prosthetic procedure of placing a full-mouth implant-supported fixed prosthesis, the combination of a healing abutment-type scan body for a digital impression and a conventional closed-mouth impression technique can reduce the number of a patient's office visits and lab procedures, tests, adjustments. In this study, we described the modified protocol for the fabrication of an implant-supported fixed complete denture. Furthermore, we compared the modified with the conventional protocol and evaluated its clinical applicability and efficiency.
\end{abstract}

Key Words: Computer-aided design; Computer-aided manufacturing; Mandible; Prosthesis

(c) This is an open-access article distributed under the terms of the Creative Commons Attribution Non-Commercial License (http://creativecommons.org/licenses/by-nc/4.0) which permits unrestricted noncommercial use, distribution, and reproduction in any medium, provided the original work is properly cited.

\section{Introduction}

For edentulous patients, implant fixed complete denture (IFCD) provides several functional and psychological advantages, such as improvement in satisfactory efficiency and pronunciation [1]. The type, passive fit, and occlusion scheme of prostheses are important to make the prostheses more secure $[2,3]$. Numerous clinical and laboratory pro- cesses are required for fabricating an IFCD in conventional procedures. Generally, impression taking with direct or indirect impression coping is performed for transferring the three-dimensional position of implant fixture and maxillomandibular relationship is recorded with record base and occlusal rim $[4,5]$. These procedures require numerous clinical and laboratory processes, which may cause inconvenience to the dentist, patient, and dental techni-

Received October 14, 2021; Revised November 3, 2021; Accepted November 5, 2021

*Corresponding author: Jung-Jin Lee, Department of Prosthodontics and Institute of Oral Bioscience, School of Dentistry, Jeonbuk National University, 567 Baekje-daero, Deokjin-gu, Jeonju 54896, Republic of Korea.

Tel: +82-63-250-2050, Fax: +82-63-250-2218, E-mail: wjdwls04@gmail.com

Copyright @ 2021, Oral Biology Research Institute 
cian. Inappropriate impression taking, improper selection of materials, errors in connection and reposition of coping and replica, and repetitive stone model fabrication can adversely affect the accuracy of the prosthesis.

The introduction of digital technology as well as computer-aided design/computer-aided manufacturing (CADCAM) facilitates efficient fabrication to produce accurate prostheses [6]. The use of digital technology can improve patient satisfaction by reducing clinical and laboratory time and the patient's number of visits [7-9]. Various attempts have been made to record the maxillomandibular relationship with digital method in the process of fabricating IFCD; however, due to the lack of accuracy, this is not clinically applicable yet $[10,11]$. Therefore, conventional procedures with an occlusal rim is commonly used for recording the maxillomandibular relationship [4]. Various laboratory processes, such as stone cast fabrication, are required, and erroneous record may be obtained because of the displacement of a recording base.

In this case report, patients with edentulous mandible were treated with IFCD and mandibular prosthesis was fabricated with two procedures; 1) conventional proceduresin which twice impressions and laboratory process need to be made with impression coping and fixture replica, 2) modified procedures-with healing abutment-type scan body and closed-mouth impression technique. Through the modified procedure, conventional processes such as impression taking with impression coping and replica and stone cast fabrication process were omitted, and the patient's number of visits and laboratory processes were reduced. This procedure reduced complicated clinical and laboratory processes and resulted in clinically acceptable treatment outcomes compared with those of conventional procedures. Therefore, this case report aimed to report such clinically acceptable treatment results.

\section{Case Description}

The patient was a 55-year-old female who underwent dental implant surgery in the mandible at a private dental clinic and came for fabricating mandibular prosthesis. At the first visit, clinical and panoramic radiographic examinations revealed complete edentulous maxilla with severe alveolar bone resorption and in the mandible, eight implants (OneQ-SL; Dentis Co., Daegu, Korea) were placed in both canines, first and second premolars, and the first molars (Fig. 1). Recording base and occlusal rim were fabricated on the diagnostic cast, and this was mounted to a articulator after recording the maxillomandibular relationship. Based on this, it was decided that the maxilla would be treated with a removable complete denture and the mandible with a hybrid fixed implant complete denture. Therefore, a temporary denture was fabricated.

One month after the second surgery, impression were taken for fabricating a definitive prosthesis. Indirect implant impression copings (DSITH45LS/DSITH55LS; Dentis Co.) were connected to the implant fixture, impressions were taken with stock tray and addition silicone impression material (Aquasil Ultra XLV/LV; Dentsply Caulk, Milford, DE, USA), and working cast was fabricated with type IV stone (GC Fujirock EP; GC Europe N.V., Leuven, Belgium) (Fig. 2).

For the accurate impression of a number of implants, which are placed in various positions and at various angulations, direct implant impression coping (DSIH45LS/ DSIH55LS; Dentis Co.) was connected to the working cast and the copings were splinted with light-curing pattern resin (JIG-GEL; Bio-den Co., Seoul, Korea) [12]. Thirty-six hours before the definitive impression was made, an individual
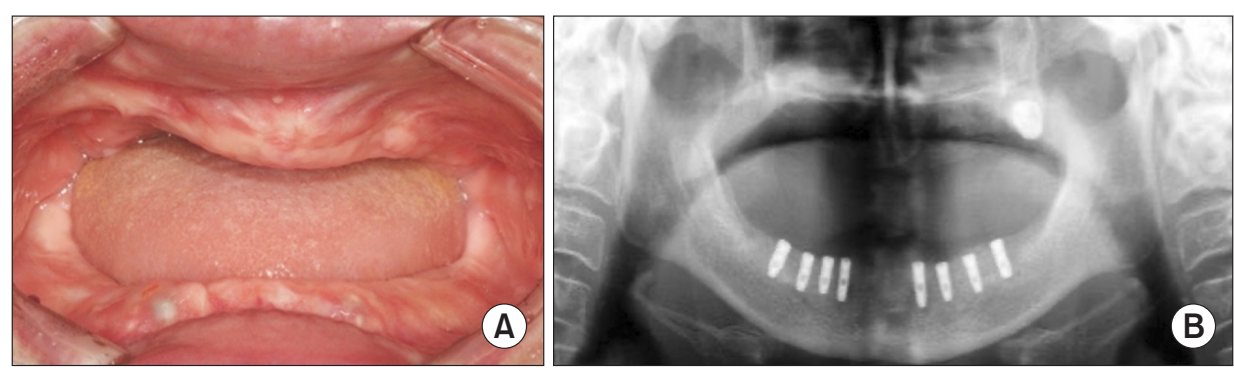

Fig. 1. Initial examination: (A) Intraoral frontal view, (B) Panoramic radiograph. 

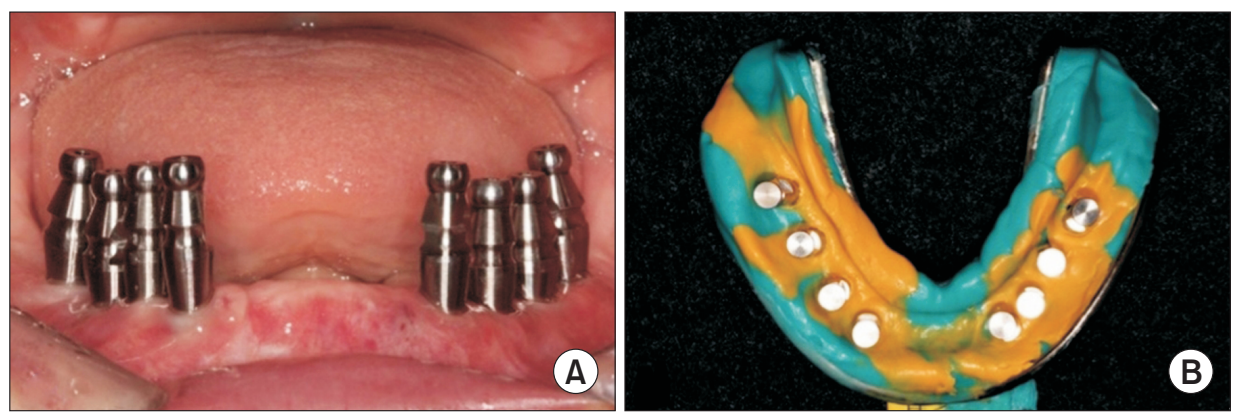

Fig. 2. (A) Indirect impression copings were connected to implants for impression. (B) Impression was made by using stock tray. Preliminary cast was fabricated to fabricate splinted direct impression copings and custom individual tray.
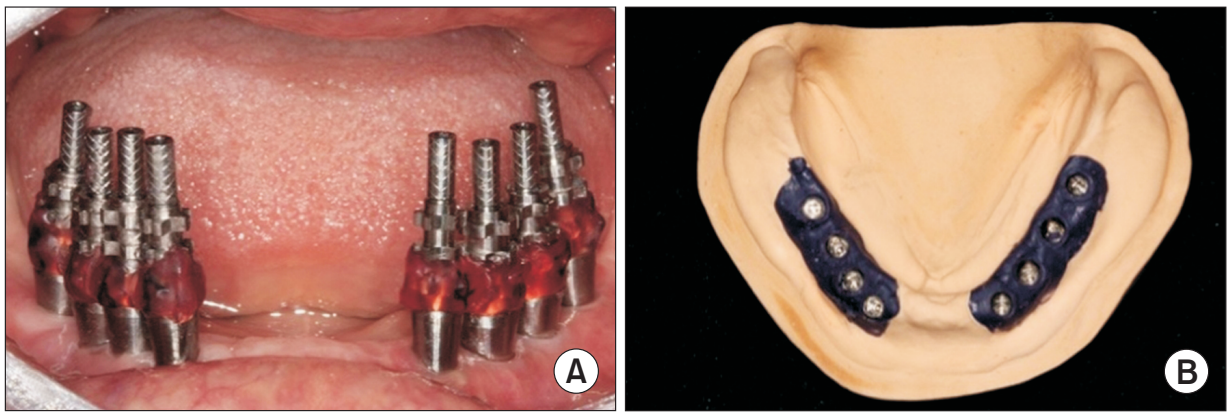

Fig. 3. (A) Splinted direct impression copings were cut, connected and splinted with pattern resin. Final impression was made with polyvinyl siloxane material using custom tray. (B) Definitive cast was fabricated with implant replica.

tray was prepared with light-curing tray resin (Lightplast Basisplatten; DreveDentamid GmbH., Unna, Germany). The secondary impression with splinted direct impression coping was taken with addition silicone impression material (Aquasil Ultra XLV/Monophase; Dentsply Caulk) (Fig. 3). A maxillary master cast was prepared after an impression was made with conventional procedures, and recording base (Lightplast Basisplatten; DreveDentamid GmbH.) and occlusal rim (Atria modeling wax; Atria Co., Seoul, Korea) were fabricated for recording the maxillomandibular relationship.

Facebow transfer and centric maxillomandibular relationship were recorded, and casts were mounted to a semi-adjustable articulator (Hanau modular articulator system 190; Whip Mix Co., Luisville, KY, USA). Considering the temporary denture and face, artificial teeth were arranged on the cast. After that, it were double scanned to design titanium custom abutment (Myplant; RaphaBio Co., Seoul, Korea) and polyetherketoneketone (PEKK) (Pekkton; Cendres+Metaux SA, Biel, Switzerland) framework with dental CAD software (Dental system 2017; 3 Shape, Copenhagen, Denmark) (Fig. 4). Polymethylmethacrylate provisional restoration (Yamahachi PMMA disk; Yamahachi Dental MFG., Gamagori, Japan) was designed, and after a

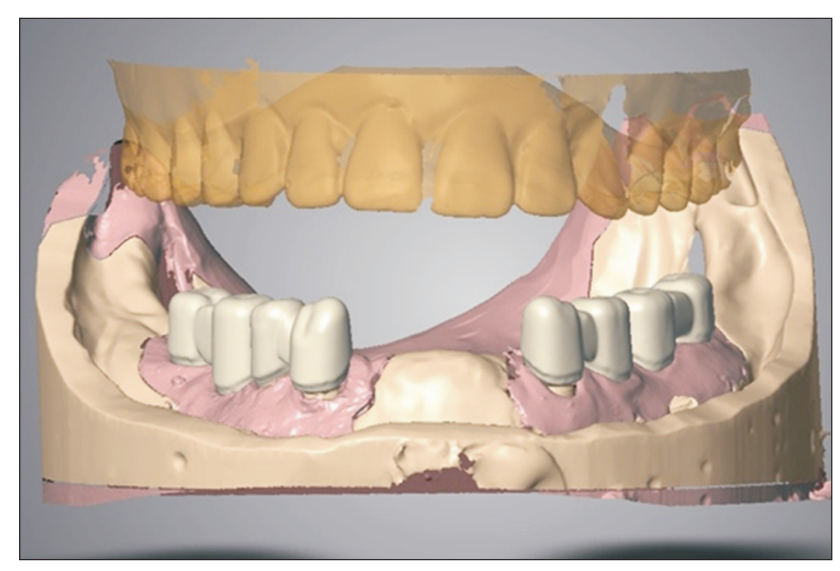

Fig. 4. Custom abutments, polyetherketoneketone framework and interim prosthesis were designed with computer-aided design software.

milling process, it was inserted in the patient with custom abutments connected to the implant and tightened with 30 Ncm (Fig. 5). After a four-month follow-up, no unsatisfactory outcome regarding the function and appearance of the implants was found. PEKK framework was fabricated and artificial teeth (Endura Anterior/Posterior; Shofu Inc., Kyoto, Japan) was arranged with maxillary removable complete denture. After try-in, definitive prostheses were fabricated with heat curing denture base resin (IvoBase Injector; IvoclarVivadent, Schaan, Liechtenstein). The mandibular 

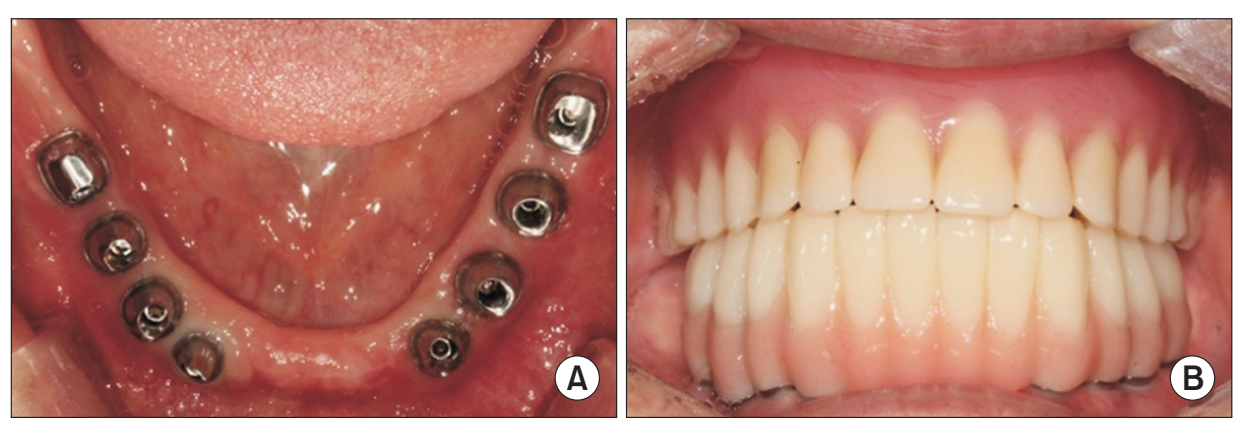

Fig. 5. (A) Titanium custom abutments were fabricated and tried-in. (B) Milled implant-supported interim prosthesis with pink gingival resin was cemented.

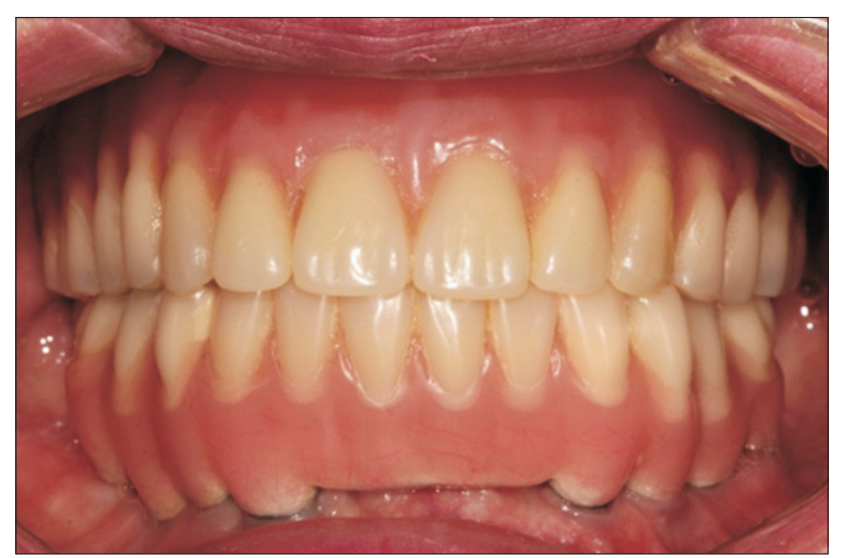

Fig. 6. Definitive prosthesis was cemented on abutments.

prosthesis was delivered with resin-modified glass ionomer cement (Fujicem2; GC Co., Tokyo, Japan) (Fig. 6).

After 12 months, the patient complained of dislocation of artificial teeth and fracture of veneered denture base resin; therefore, re-fabrication of monolithic zirconia prosthesis was planned. The old mandibular prosthesis was removed, and healing abutment-type scan body (Healoc abutment; Truabutment Inc., Irvine, CA, USA) was connected to the fixture corresponding to implant diameter and gingival height. A preliminary impression was taken with an irreversible hydrocolloid impression material, and a preliminary cast was fabricated (Fig. 7). On the preliminary cast, recording base (Lightplast Basisplatten; DreveDentamid $\mathrm{GmbH}$.) and occlusal wax rim (Atria modeling wax; Atria Co.) were fabricated. The tentative vertical dimension and maxillomandibular relationship were determined in consideration of the existing prostheses. Border molding (Virtual heavy body; IvoclarVivadent) was done, an impression was taken with closed-mouth impression technique with a recording base and addition rubber impression material
(Aquasil XLV; Dentsply Caulk), and the maxillomandibular relationship was recorded with bite registration materials (O-Bite; DMG, Hamburg, Germany) (Fig. 8). After a definitive cast was made with type IV stone (GC Fujirock EP; GC Europe N.V.), snap-on type scan body was connected to Healoc abutment of the cast and scanned with a laboratory scanner (D2000; 3 Shape A/S). With the CAD software (Dental system 2017; 3 Shape), custom titanium abutment (DS titan abutment; Truabutment Inc.) and temporary prostheses were designed and fabricated through milling procedure. The abutments were connected to the implants with 30-Ncm tightening torques. After try-in and occlusal adjustment, the temporary prostheses were set (Fig. 9).

Function and oral hygiene ability evaluated during threemonth follow-up period, and no pathologic sign was observed. The previously designed monolithic zirconia prosthesis (Pre-shade blank MT A3; Liaoning Upcera Co., Guangdong, China) was fabricated with computer-aided manufacturing (ARUM 5X-100; Doowon ID., Daejeon, Korea). After try-in and occlusal adjustment, the prosthesis was set with resin-modified glass ionomer cement (Fujicem2; GC Co.) (Fig. 10). Oral hygiene was observed at the patient's visit over seven months after the insertion of the prosthesis, and the patient expressed her satisfaction with the function and appearance of the prosthesis. Furthermore, no complications, such as mobility of prosthesis and cement loss, were observed.

\section{Discussion}

In this study, for a mandibular edentulous patient, a hybrid fixed complete denture was fabricated with conventional procedures, and custom abutment and zirconia 

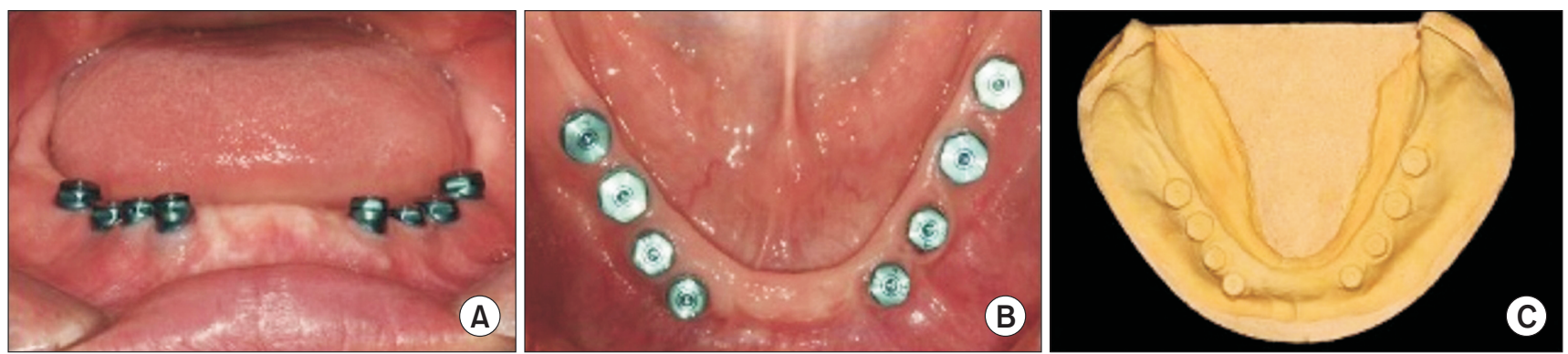

Fig. 7. Clinical image of Healoc abutment-type scan body in place after removal of healing abutments: (A) Frontal view, (B) Occlusal view, (C) Working stone cast for making a record base and a wax occlusal rim.
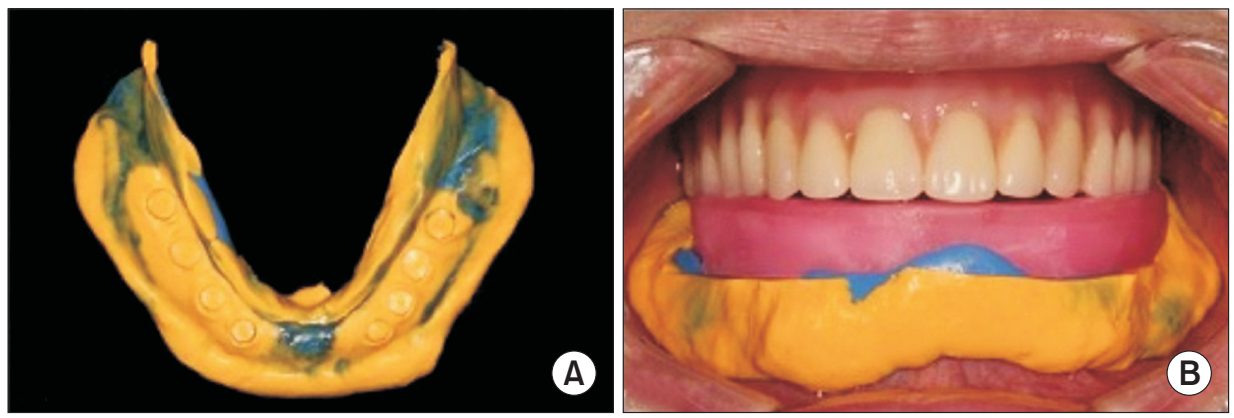

Fig. 8. (A) Border molding and final impression were taken with polyvinyl siloxane. (B) Vertical dimension and final impression were taken at same time.
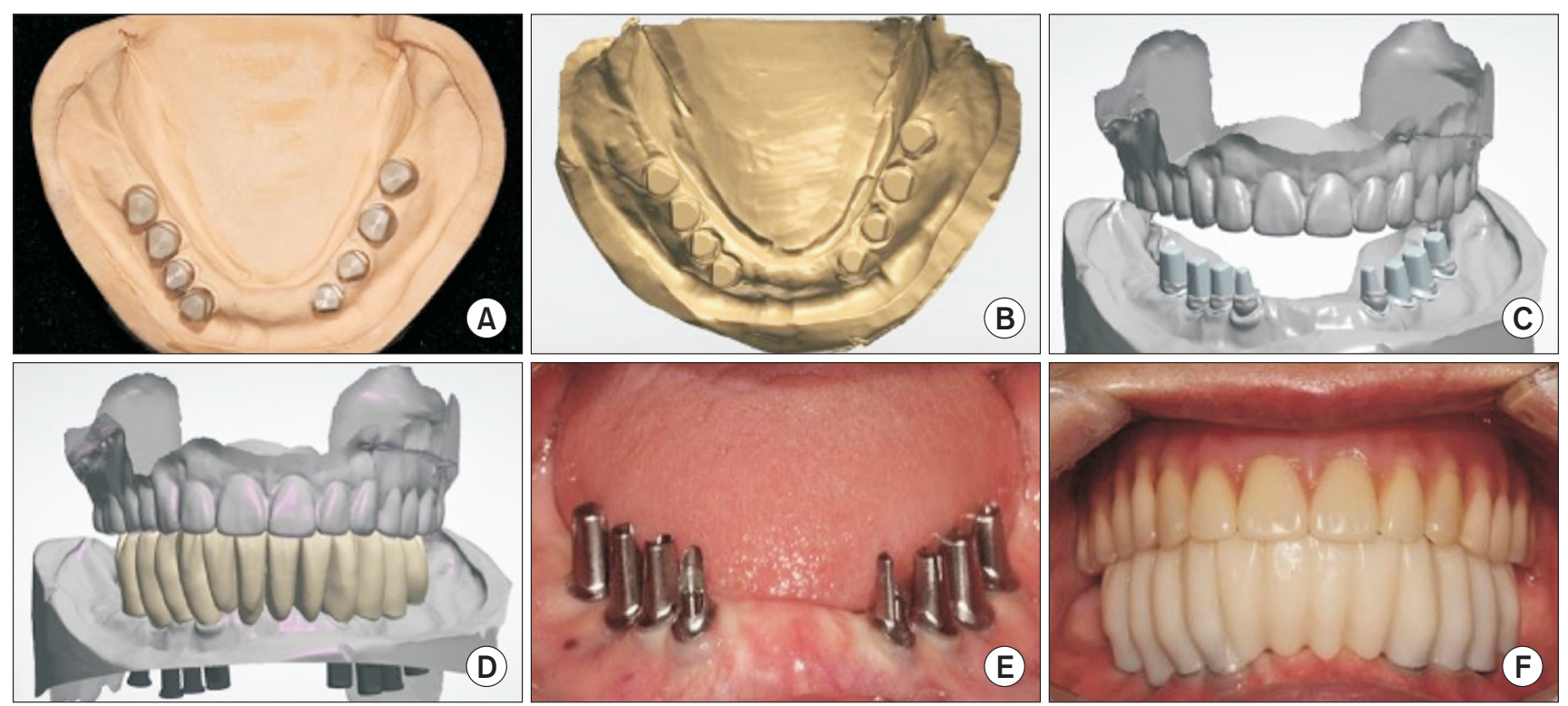

Fig. 9. (A) Snap-on scan bodies were connected to Healoc abutment of definitive cast. (B) Definitive cast and scan bodies were scanned with laboratory scanner. (C) Computer-aided design of individualized abutment. (D) Computer-aided design of final prosthesis. (E) Custom abutments try-in. (F) Provisional prosthesis try-in.

prosthesis were fabricated with a modified procedure with healing abutment-type scan body and closed-mouth impression techniques. For the passive fit of IFCD, impressions need to be made several times and stone cast fabrica- tion is needed [13]. It is also important to record proper vertical dimension and maxillomandibular relationship for the restoration of function and esthetics in a patient. With conventional procedure, they can be recorded with record- 

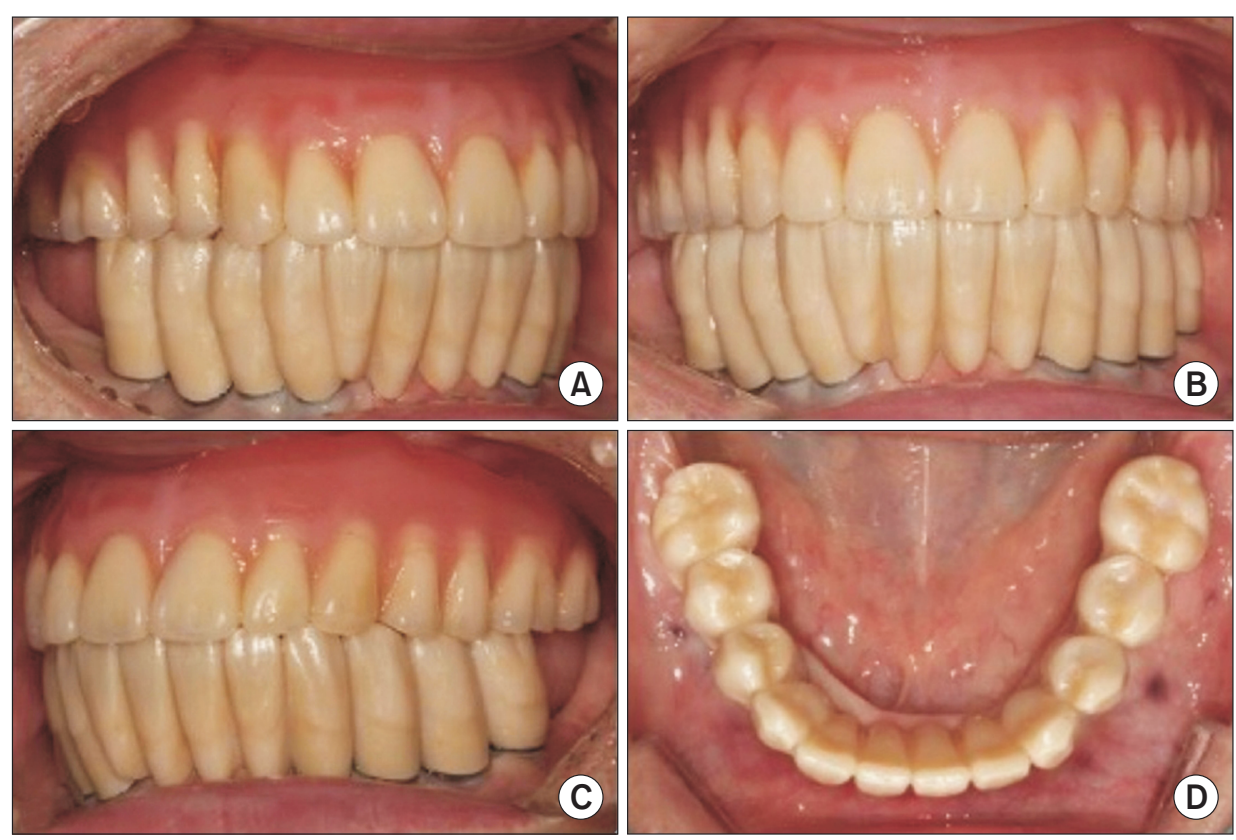

Fig. 10. Definitive prostheses: (A) Right view, (B) Frontal view, (C) Left view, (D) Occlusal view of lower prosthesis.

ing base and occlusal rim, and after the arrangement of artificial teeth and through trial adaptation, lip support and pronunciation can be confirmed [4]. This can cause some inconveniences; numerous clinical and laboratory processes are required, patients need to visit frequently. In addition, repeated clinical and laboratory processes can result in some errors.

The procedure with healing abutment-type scan body and closed-mouth impression technique used in this study has many advantages. Healing abutment-type scan body of which the height is less compared with conventional impression coping was connected. The definitive impression with closed-mouth impression technique was taken, and the maxillomandibular relationship was recorded at the same time. By using healing abutment-type scan body, unlike conventional impression taking, healing abutments do not need to be disconnected or reconnected repeatedly; for that reason, patients do not feel any discomfort, and time for clinical process can be saved. The patient's number of visit is also reduced as closed-mouth impression technique allows impression making and maxillomandibular relationship recording to be done at the same time. Furthermore, custom abutments and definitive prostheses were fabricated without a cast with impression copings and replica, thus reducing the chance of errors due to replica connection and coping repositioning [14]. Moreover, this procedure is economically advantageous as it does not require numerous impression copings and replicas [15-17]. When hybrid prosthesis was fabricated with conventional procedure, the patient had visited seven times and eight-stage laboratory process and sixteen impression copings and replicas were needed. However, when prosthesis was fabricated with the proposed procedure, the patient visited only once for impression taking and recording the maxillomandibular relationship and custom abutment and temporary prosthesis were fabricated immediately. In addition, the relation recorded with the impression body, which was made with closed-mouth impression technique, contributed to higher stability of recording base and occlusal rim than the conventional procedure, which resulted in the reduced chance of occlusal errors due to the recording errors of the maxillomandibular relationship.

As implant prostheses were fabricated without a stone cast with implant replica in this study, it had some limitations that the passive fit between abutment and prosthesis cannot be directly checked. However, this problem can be solved by fabricating a cast with stereolithography, which has advanced in recent years. If the procedure proposed in this case report can be scientifically studied more, accurate prostheses would be fabricated with more simplified process. 


\section{Acknowledgements}

This paper was supported by Fund of Biomedical Jeonbuk National University Hospital.

\section{Conflicts of Interest}

The authors declare that they have no competing interests.

\section{ORCID}

\author{
Ju-Mi Park \\ https://orcid.org/0000-0003-1910-1525 \\ Yeon-Hee Park \\ https://orcid.org/0000-0002-9183-9244 \\ Jung-Jin Lee \\ https://orcid.org/0000-0002-7381-5230
}

\section{References}

1. Misch CE. Contemporary implant dentistry. 3rd ed. St. Louis: Mosby; 2008;17-20.

2. Sones AD. Complications with osseointegrated implants. J Prosthet Dent 1989;62:581-585. doi: 10.1016/0022-3913 (89)90084-x.

3. Jemt T, Lindén B, Lekholm U. Failures and complications in 127 consecutively placed fixed partial prostheses supported by Brånemark implants: from prosthetic treatment to first annual checkup. Int J Oral Maxillofac Implants 1992;7:40-44.

4. Sadowsky SJ. The role of complete denture principles in implant prosthodontics. J Calif Dent Assoc 2003;31:905909.

5. Savabi O, Nejatidanesh F. Interocclusal record for fixed implant-supported prosthesis. J Prosthet Dent 2004;92:602603. doi: 10.1016/j.prosdent.2004.09.004.

6. Nayar S, Mahadevan R. A Paradigm shift in the concept for making dental impressions. J Pharm Bioallied Sci 2015;7 Suppl 1:S213-S215. doi: 10.4103/0975-7406.155910.

7. Christensen GJ. Impressions are changing: deciding on conventional, digital or digital plus in-office milling. J Am Dent Assoc 2009;140:1301-1304. doi: 10.14219/jada.archive.2009.0054.

8. Pyo SW, Park YB, Kim JH, Moon HS, Lee KW. Maxillary anterior all ceramic restoration using digital impression and CAD/CAM. J Korean Acad Prosthodont 2011;49:263-269. doi: 10.4047/jkap.2011.49.3.263.

9. Hong YS, Park EJ, Kim SJ, Kim MR, Heo SJ, Park JM. Customized abutment and screw-type implant prostheses after cementation based on the digital intra-oral impression technique. J Korean Acad Prosthodont 2012;50:67-73. doi: 10.4047/jkap.2012.50.1.67.

10. Patil PG, Nimbalkar-Patil S. Maxillomandibular relationship record for implant complete mouth rehabilitation with elastomeric material and facial surface index of existing denture. J Indian Prosthodont Soc 2015;15:337-341. doi: 10.4103/0972-4052.161568.

11. Li W, Xie Q, Wang Y, Sun Y. A pilot study of digital recording of edentulous jaw relations using a handheld scanner and specially designed headgear. Sci Rep 2018;8:8975. doi: 10.1038/s41598-018-27277-5.

12. Moreira AH, Rodrigues NF, Pinho AC, Fonseca JC, Vilaça JL. Accuracy comparison of implant impression techniques: a systematic review. Clin Implant Dent Relat Res 2015;17 Suppl 2:e751-e764. doi: 10.1111/cid.12310.

13. Wee AG. Comparison of impression materials for direct multi-implant impressions. J Prosthet Dent 2000;83:323331. doi: 10.1016/s0022-3913(00)70136-3.

14. Papaspyridakos P, Chen CJ, Chuang SK, Weber HP, Gallucci GO. A systematic review of biologic and technical complications with fixed implant rehabilitations for edentulous patients. Int J Oral Maxillofac Implants 2012;27:102-110.

15. Lee H, So JS, Hochstedler JL, Ercoli C. The accuracy of implant impressions: a systematic review. J Prosthet Dent 2008;100:285-291. doi: 10.1016/S0022-3913(08)60208-5.

16. Derhalli M. The digitalizing of implant dentistry: a clinical evaluation of 15 patients. Compend Contin Educ Dent 2013;34:192-196.

17. Abduo J, Chen C, Le Breton E, Radu A, Szeto J, Judge $\mathrm{R}$, Darby I. The effect of coded healing abutments on treatment duration and clinical outcome: a randomized controlled clinical trial comparing encode and conventional impression protocols. Int J Oral Maxillofac Implants 2017;32:1172-1179. doi: 10.11607/jomi.5386. 